\title{
Energy-Efficient Runtime Management of Heterogeneous Multicores using Online Projection
}

\author{
STAVROS TZILIS, PEDRO TRANCOSO, and IOANNIS SOURDIS, \\ Chalmers University of Technology
}

\begin{abstract}
Heterogeneous multicores offer flexibility in the form of different core types and Dynamic Voltage and Frequency Scaling (DVFS), defining a vast configuration space. The optimal configuration choice is not always straightforward, even for single applications, and becomes a very difficult problem for dynamically changing scenarios of concurrent applications with unpredictable spawn and termination times and individual performance requirements. This article proposes an integrated approach for runtime decision making for energy efficiency on such systems. The approach consists of a model that predicts performance and power for any possible decision and low-complexity heuristics that use this model to evaluate a subset of possible decisions to choose the best. The model predicts performance by projecting standalone application profiling data to the current status of the system and power by using a set of platform-specific parameters that are determined only once for a given system and are independent of the application mix. Our approach is evaluated with a plethora of dynamic, multi-application scenarios. When considering best effort performance to be adequate, our runtime achieves on average $3 \%$ higher energy efficiency compared to the powersave governor and $2 \times$ better compared to the other linux governors. Moreover, when also considering individual applications' performance requirements, our runtime is able to satisfy them, giving away $18 \%$ of the system's energy efficiency compared to the powersave, which, however, misses the performance targets by $23 \%$; at the same time, our runtime maintains an efficiency advantage of about $55 \%$ compared to the other governors, which also satisfy the performance constraints.
\end{abstract}

CCS Concepts: • Computer systems organization $\rightarrow$ Embedded software; System on a chip; $\bullet$ Hardware $\rightarrow$ Power estimation and optimization; $•$ Theory of computation $\rightarrow$ Design and analysis of algorithms;

Additional Key Words and Phrases: Heterogeneous multicores, dynamic voltage and frequency scaling, runtime management, energy efficiency

\section{ACM Reference format:}

Stavros Tzilis, Pedro Trancoso, and Ioannis Sourdis. 2019. Energy-Efficient Runtime Management of Heterogeneous Multicores using Online Projection. ACM Trans. Archit. Code Optim. 15, 4, Article 63 (January 2019), 26 pages.

https://doi.org/10.1145/3293446

This research was supported by the European Research Council (ERC) under the MECCA project (Contract No. 340328) Authors' addresses: S. Tzilis, P. Trancoso, and I. Sourdis, CSE Department, Chalmer University of Technology, 41296 Gothenburg Sweden; emails: \{tzilis, ppedro, sourdis\}@chalmers.se.

Permission to make digital or hard copies of all or part of this work for personal or classroom use is granted without fee provided that copies are not made or distributed for profit or commercial advantage and that copies bear this notice and the full citation on the first page. Copyrights for components of this work owned by others than the author(s) must be honored. Abstracting with credit is permitted. To copy otherwise, or republish, to post on servers or to redistribute to lists, requires prior specific permission and/or a fee. Request permissions from permissions@acm.org.

(C) 2019 Copyright held by the owner/author(s). Publication rights licensed to ACM.

1544-3566/2019/01-ART63

https://doi.org/10.1145/3293446 


\section{INTRODUCTION}

Multicore architectures often offer heterogeneity and Dynamic Voltage and Frequency Scaling (DVFS). This flexibility provides knobs for trading performance for energy and vice versa thereby improving system efficiency. Commercial examples of such, single-ISA, architectures include the Samsung Exynos [21] and Qualcomm Snapdragon [19] processors. They employ multiple types of cores (power-efficient and high performance), and multiple voltage-frequency pairs per cluster of cores. In this manner, they can potentially adapt to the fluctuating system workload and its needs at runtime. Such runtime variations stem from the number, type, and performance requirements of the workloads running at any given time on the system. Adapting the system to these dynamic changes can balance smooth application operation and performance with energy efficiency.

In this context, choosing the optimal system configuration is often not straightforward. Executing an application on a power-efficient core might prolong execution enough to reduce energy efficiency, while choosing a high-performance core poses the opposite risk-an increase of power consumption outweighing performance gains. DVFS adds more complexity to this problem, as it is unclear how a high-performance core at a low frequency and a power-efficient core at a high frequency compare in terms of energy efficiency [26]. Interference between applications sharing system resources, i.e., levels of the memory hierarchy, makes the runtime decision even harder. In conclusion, today's Chip Multiprocessors (CMPs) offer great potential for performance and energy efficiency, which is not fully utilized due to the difficulty of choosing optimal configurations [6, $11,13,28]$.

In the past, the challenge of the system load being dynamic and unpredictable has been answered in the following two ways. Some solutions make an one-off decision whenever an event happens, such as an application spawning or termination. They predict the impact of such events using offline application characterization coupled with existing system monitoring information. Subsequently, they choose a new system configuration aiming to maximize energy efficiency, requiring little to no corrective action until the next event. However, predicting the performance of different applications running concurrently and competing for shared resources, is extremely complex. This leads to other solutions that avoid this challenge by following a trial-and-error approach. They begin with a naïve, even random, placement of applications and subsequently use online monitoring to gradually converge to a more energy-efficient system configuration, i.e., choice of cluster frequencies and/or application placements [5, 7]. Such approaches may require a long time to converge to a satisfactory configuration and may suffer from poor scaling of response time when system size increases.

In this work, elements of both above approaches are combined. In response to an event (application spawning or termination), the space of possible system configurations is partially searched, predicting the energy efficiency of different options and selecting the best one. Subsequently, this decision can be revisited when more accurate system monitoring information becomes available. However, our main focus is on the initial prediction and decision step to minimize any need for subsequent adjustments. Gradually adapting the system can be a slow and costly process, especially when the initially selected configuration is inefficient. Thus, it is important that the first response to an event is an efficient one.

The two main challenges of this approach are (i) creating a model that accurately predicts performance and power for arbitrary candidate system configurations and (ii) developing a fast and scalable decision algorithm, since it is not practical to evaluate all possible configurations at runtime. In an attempt to face these challenges, this article makes the following novel contributions:

- A model capable of estimating all running applications' performance and power, for every possible decision to be taken whenever an event (application spawning or termination) 
Table 1. Categorization of Existing Works with Respect to Various Aspects

\begin{tabular}{|l||c|c||c|c|c||c|c|c||l|}
\hline & $\begin{array}{c}\text { Mult. } \\
\text { App. }\end{array}$ & $\begin{array}{c}\text { Perf. } \\
\text { Req. }\end{array}$ & \multicolumn{3}{c||}{$\begin{array}{c}\text { Energy-saving } \\
\text { Techniques }\end{array}$} & \multicolumn{3}{c||}{ Decision guided by } & \multicolumn{1}{|c|}{ Decision Strategy } \\
\hline & & & DVFS & DPM & Placement & Profiling & Monitoring & Prediction & \\
\hline \hline Liu et al. [14] & $\boldsymbol{V}$ & & & & $\boldsymbol{V}$ & & $\boldsymbol{V}$ & $\boldsymbol{V}$ & Heuristic, $O\left(n^{2}\right)$ \\
\hline NORNIR [5] & & $\boldsymbol{V}$ & $\boldsymbol{V}$ & & $\boldsymbol{V}$ & & $\boldsymbol{V}$ & $\boldsymbol{V}$ & Runtime model refinement \\
\hline SPARTA [7] & $\boldsymbol{V}$ & $\boldsymbol{V}$ & $\boldsymbol{V}$ & & $\boldsymbol{V}$ & & $\boldsymbol{V}$ & $\boldsymbol{V}$ & Heuristic, $O\left(n^{2}\right)$ \\
\hline Aalsaud et al. [1] & & & $\boldsymbol{V}$ & $\boldsymbol{V}$ & & $\boldsymbol{V}$ & & $\boldsymbol{\checkmark}$ & Heuristic, $O(n)$ \\
\hline DyPO [9] & & & $\boldsymbol{V}$ & $\boldsymbol{V}$ & & $\boldsymbol{V}$ & $\boldsymbol{V}$ & & Matching profiled status \\
\hline \hline Our approach & $\boldsymbol{V}$ & $\boldsymbol{V}$ & $\boldsymbol{V}$ & $\boldsymbol{(})$ & $\boldsymbol{V}$ & $\boldsymbol{V}$ & $\boldsymbol{V}$ & $\boldsymbol{V}$ & Heuristics $(O(n * \log n))$ \\
\hline
\end{tabular}

happens. The model uses a combination of (i) online performance monitoring, (ii) online projection of offline-acquired application performance characteristics, and (iii) static platform characterization parameters, to predict the result of candidate decisions.

- Three runtime heuristics that use the above model to choose a new system configuration whenever an event happens. They select a small number of candidate solutions to evaluate with the prediction model and make a decision that respects all applications' performance requirements while maximizing energy efficiency of the system in terms of IPS/Watt.

The rest of the article is organized as follows: Section 2 summarizes related work. Section 3 describes our prediction model. Section 4 presents our decision algorithms and analyzes them in terms of complexity. Section 5 contains the experimental evaluation. Section 6 discusses some alternatives to and limitations of the proposed approach. Last, Section 7 recaps the main takeaway points of the whole study.

\section{RELATED WORK}

There exists a substantial amount of research on energy-efficiency in heterogeneous CMPs. We hereby attempt to identify the main features that differentiate these works and point out our novelty with respect to these features. The most representative works are categorized in Table 1.

An important categorization of existing works is with respect to the application scenario they focus on. Some focus almost exclusively on single-application scenarios [4, 5, 9], while others consider concurrent execution of multiple applications [2, 7]. A representative example is SPARTA by Donyanavard et al. [7]. SPARTA proposes a task allocation strategy handling various mixes of single- or multi-threaded applications, treating threads of the same application as separate tasks. We consider this multiple-application scenario more interesting, as it is closer to real-life use cases such as mobile devices. We aim to maximize overall system Instructions per Second per Watt (IPS/Watt) for concurrent execution. While we do not use multi-threaded versions, we allow multiple instances of the same application to exist simultaneously. Alongside the number and type of applications, another aspect of the considered scenario is the existence of requirements for individual applications. On the currently trending cluster-based architectures, a single application that needs to maintain a minimum performance can impact the energy efficiency of a whole cluster, which is a significant part of the whole system. Thus, it is important that the runtime manager does not raise the frequency higher than needed to fulfill the performance requirement, as this typically results to lower energy efficiency. Similar to References $[5,7,17]$, we consider this aspect of runtime management important, thus we equip our algorithms with the ability to take care of 
individual applications' requirements, something that is not present in works such as References $[1,9,14]$.

Another important component is the combination of techniques utilized to extract energy savings. The three main alternatives are: (i) Dynamic Voltage and Frequency Scaling (DVFS) [12], which focuses on selecting cluster frequencies; (ii) Dynamic Power Management (DPM) [3], which allows for components that are not currently used to be switched off; (iii) application placement (allocation) [23], which involves the decision of which type of core every application executes on. Existing works utilize various mixes of the three techniques. Works with more focus on singleapplication scenarios focus on DPM, seeking the optimal configuration of active and inactive cores [9]. On top of this, some strategies decide the application placement and predict the decision of the frequency governor without taking control of the DVFS aspect [7, 22], while others also control voltage and frequency, but still evaluate their approach for single applications $[4,5$, 24]. In this work, we fully control DVFS and application placements, while at the same time allowing the DPM governor to turn off idle cores. Thus, our approach covers all three techniques of energy saving, as the DPM decision is implicit given a specific placement of applications: cores that do not currently execute an application can be set to an idle state.

The central component of every strategy, is the set of information considered for making decisions. In the context of energy efficiency runtime management for heterogeneous CMPs, we identify three possible sources of information: (i) offline application profiling; (ii) online, runtime performance monitoring; (iii) predictions performed with a suitable model. The three sources are explained separately in each of the following three paragraphs.

Many approaches utilize application profiling data obtained offline. For instance, DyPO by Gupta et al. [9] uses extensive offline application profiling to create a library of possible system statuses with respect to important metrics such as instructions retired, memory accesses, and cache misses. For each of these statuses, the optimal configuration is identified and stored. At runtime, the system manager measures the same features online, to identify the library entry that is the closest to the current status and apply the configuration that offline profiling dictates to be the best. We agree that offline-acquired performance characteristics can guide a decision at runtime and consider reasonable to use profiling to extract this information. However, we identify and solve a scalability concern of such strategies: The offline nature of profiling creates a misconception of unlimited available time, which is not realistic. As an example, for a Samsung Exynos board with only four ARM A7 and four ARM A15 cores, there are 4,004 possible configurations. DyPO [9] tackles this problem by selecting a representative subset of these configurations but unfortunately does not propose a scalable extension of this strategy to larger systems. Considering that core count tends to increase and that an unpredictably high number of applications can run on a system during its lifetime, we go one step further and propose a scalable strategy for choosing configurations for offline application characterization. Our strategy requires a total amount of runs that is linear with respect to the number of cores. To further ensure the scalability of this offline stage, we limit ourselves to standalone profiling of each application, regardless of the various mixes that can appear at runtime. As will be explained, the information acquired during profiling is adapted at runtime using our online projection technique.

Regardless of whether or not offline profiling is used, most approaches rely on online performance monitoring, especially works such as Reference [7], that target dynamic multi-application scenarios, as we also do in this article. Online monitoring is essential for capturing varying performance characteristics of individual applications. Such performance variations are caused by the dynamic nature of the system workload: spawning and termination of applications affect the contention for shared resources, which in turn affects other applications' performance. Taking compact, static, application-specific offline profiling information (see previous paragraph) and 
projecting it to the dynamic, application mix-dependent status of the system, as it is measured by online performance monitoring, is one of the core contributions of our work.

Although most strategies involve some kind of modelling of performance and power [18], there is an important distinction in whether or not this model is used to perform explicit predictions, which in turn guide the decisions. For instance, NORNIR proposed by De Sensi et al. [5] is a representative example of a strategy utilizing explicit predictions. In NORNIR, a newly spawned application, of which no prior knowledge is assumed, is first tried on a sequence of different configurations to refine a prediction model during a calibration phase. The refined model is subsequently used to choose a close-to-optimal configuration for the steady phase. During this final configuration selection step, explicit routines predict application performance and system power for various candidate configurations, and the configuration with the highest predicted efficiency is selected. On the contrary, in DyPO [9], the runtime decision for a system configuration is driven by identifying an offline-profiled system status that is similar to the current runtime status. A configuration that was efficient for the profiled status, is expected to also be efficient in the current similar runtime situation, without explicit prediction of particular metrics for performance or power. Furthermore, if we scrutinize approaches that use explicit prediction, we can identify qualitative differences among their prediction models. The prediction model of Reference [7] is based on the lightweight but coarser-grain approach of simply categorizing (binning) applications with respect to a number of compute- and memory-boundedness criteria. In Reference [1], the prediction model is workload-specific, requiring retraining every time a new application mix appears. In NORNIR [5], as explained above, online calibration (refinement) is performed instead of retraining, making it a more realistic solution for dynamic, unpredictable scenarios. Unfortunately, the calibration phase has to attempt an unbounded number of non-optimal configurations, thus it is still difficult to predict its effect on the end-to-end efficiency. We achieve performance prediction by projecting offline profiling data to the online-monitored current system status and power prediction through characterization of the actual board, that produces a static, platform-specific power model involving no application-specific parameters. As such, our models perform fine-grain performance and power prediction, without the need for retraining or runtime refinement.

Last, existing works differ with respect to the strategy used to make runtime decisions. Liu et al. [14] established that low-complexity heuristics can produce solutions very close to the optimal, in time two to three orders of magnitude lower than optimal solvers. However, their heuristic's execution time scales quadratically with the number of cores. The allocator proposed in SPARTA [7] also includes an iterative step, resulting in an execution time that explodes when going from a 32-core system to a 64- and 128-core system. However, Aaslaud et al. propose a linear-complexity algorithm in Reference [1]. Unfortunately, the fact that this heuristic is based on a model that needs to be retrained for each application mix, is a major drawback for a runtime optimization strategy, as it reduces its applicability when it comes to unpredictable, dynamically changing scenarios. It is clear, however, that the complexity of the proposed heuristics has to be carefully chosen, to trade well between solution quality and execution time. We propose different heuristics with complexity of at most $O(n * \log n)$.

Table 1 summarizes the most representative existing works with respect to the aspects described above. The first column, "Mult. App.," indicates whether or not the proposed approach can support dynamic, unpredictable scenarios of multiple concurrent applications, while the next column, "Perf. Req.," states whether or not it satisfies performance requirements of individual applications. The next three columns ("Energy-saving Techniques") indicate which of the identified energy-saving knobs are used. A parenthesized tick mark means that the technique is utilized but not explicitly controlled. The next three columns ("Decision guided by") indicate what kind of 
information is used to guide runtime decisions. Last, the "Decision Strategy" column mentions the runtime strategy used and its complexity, where applicable.

In summary, the approach proposed by this article: finds a sweet spot for utilizing both offline profiling and online monitoring information, keeping profiling scalable and confined to single applications, while using monitoring and online projection to incorporate the dynamic, unpredictable characteristics of the current application mix; decouples performance and power prediction, performing the former as described in the previous point, and the latter with a model trained only once for a specific platform, since it involves only architecture-specific parameters; targets a realistic scenario, as it considers concurrent execution of arbitrary application mixes and takes care of individual applications' performance requirements.

\section{PERFORMANCE AND POWER PREDICTION}

This section provides a detailed description of our model, used to predict the performance and power impact of runtime decisions before applying them. Application ${ }^{1}$ performance is predicted by projecting profiling information to the current system status. In turn, the predicted performance is used as input to our power model. Our approach revolves around different performance metrics, namely retired instructions and accesses of all levels of the memory hierarchy, per unit of time. Thus, on a system with two levels of cache, such as the ODROID XU3 [20] (see upper part of Figure 1, labelled "Hardware"), we are interested in the following metrics:

- Retired instructions per second (IPS)

- L1 data cache accesses per second (L1aps)

- LLC (L2) accesses per second (LLCaps)

- DRAM accesses per second (DRAMaps)

Predicting future values of these performance metrics is complicated by the dynamically changing system status. We demonstrate this with a very simple motivating example. We run the SP and CG benchmarks of the NAS suite [15], on a cluster of big (A15) cores of an ODROID XU3 board, in two different setups at the same frequency: first, standalone (one by one) and then in parallel (on different cores of the same cluster, but simultaneously). We observe that the performance of each degrades by almost $20 \%$ when they execute in parallel because of the competition for shared resources (L2 cache and DRAM). In literature, this challenge is tackled by using prediction models that involve parameters specific to each application mix [1]. This, in turn, creates the need for retraining or refining the model, every time the application mix changes.

To answer the above drawback, we introduce the technique of online projection of profiling information. The core idea of projection is that performance characteristics acquired by profiling a standalone application offline, can be combined with online performance monitoring at runtime, to extrapolate the application's behavior when it runs concurrently with other applications. The offline profiling determines the individual characteristics of each single application, while the online monitoring of the system's current status incorporates the effect of competition for shared resources, eliminating the need for model retraining. More specifically, when profiling each application, we force it to compete with other instances of itself for shared system resources, such as the LLC and the DRAM. We vary the amount of competition and record how the application's performance varies as a result. At runtime, we use performance counters to obtain awareness of the current level of competition for shared resources in the system. When the profiled application

\footnotetext{
${ }^{1}$ Henceforth, the term "application" refers to single-threaded applications, as this is the case we target in this article, as explained in Section 2.
} 


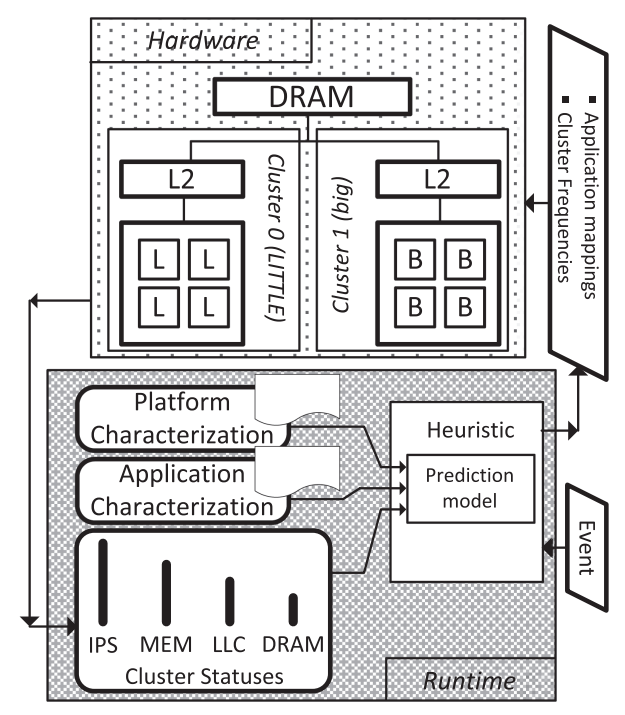

Fig. 1. The ODROID XU-3 hardware and our runtime.

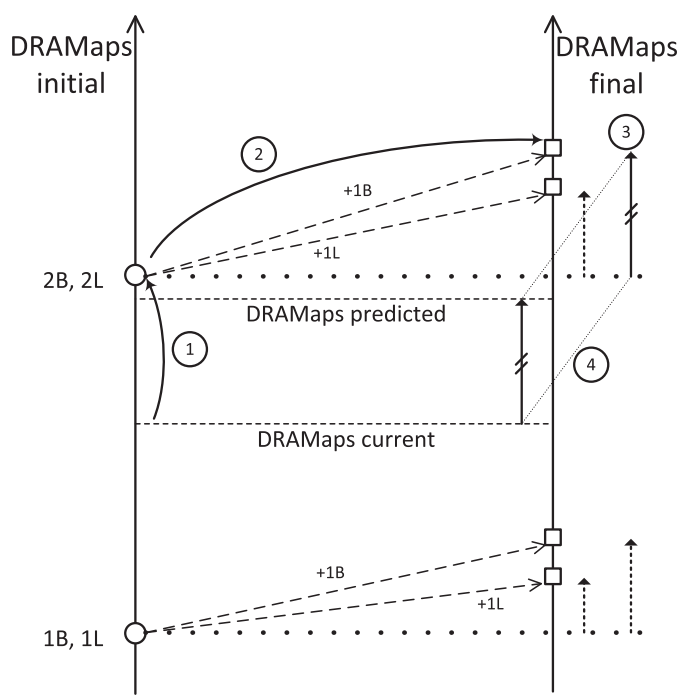

Fig. 2. Online projection for performance prediction.

spawns, we match the online measurement to the closest profiled value and use it as a starting point to predict its performance in the current situation.

The above process is illustrated in Figure 2. It involves profiling an application in several different configurations-how these are determined will be explained shortly. For now, to keep this example simple, let us suppose that we only use two configurations: two and four instances of the application running in parallel, evenly divided between the big (B) and little (L) cluster of an ODROID XU3, resulting to the initial states $(1 \mathrm{~B}, 1 \mathrm{~L})$ and $(2 \mathrm{~B}, 2 \mathrm{~L})$, listed and marked with circles on the "DRAMaps initial" axis. We measure total DRAM accesses per second (DRAMaps) for each of these scenarios. Then, for each scenario, we spawn one extra instance on the big cluster and record the change in the DRAMaps metric. We do the same for one extra instance on the little cluster. These final states are marked with squares on the "DRAMaps final" axis, at the ends of the " $+1 \mathrm{~B}$ " and " $+1 \mathrm{~L}$ " arrows. The upward-pointing arrows at the right side of the picture represent the DRAMaps increment for each final state. At runtime, we monitor the total amount of system DRAMaps online-marked with a horizontal dashed line labelled "DRAMaps current." When the specific application is spawned, we find the initial state closest to the current DRAMaps value, in this example $(2 \mathrm{~B}, 2 \mathrm{~L})$ (1). This is called resembling state, since it is the one closest to the current system state with respect to the particular performance metric. Then, if we want to place the application on the big cluster, we follow the " $+1 \mathrm{~B}$ " line and retrieve the DRAMaps value of this final state(2), which we call projected state, since it represents the impact of the spawned application on the system. To quantify the projection, we use the difference of DRAMaps between projected and resembling state, which in this example is represented by the solid upwards-pointing arrow (3). Adding this difference to "DRAMaps current" gives us the predicted value of DRAMaps ("DRAMaps predicted") (4), if we proceed with the placement of the application on a big core.

Aside the competition for shared resources, application performance is also affected by the operating frequency. To avoid profiling for every supported frequency, the frequency effect on performance is considered by estimating a scaling factor for each application. This factor depends on the compute- versus memory-intensiveness of the application and will be further explained in Section 3.1.2. Consequently, profiling information is used to characterize each application in terms 
of compute- versus memory-boundedness. At runtime, we use this information to predict the impact of frequency on application performance. Our profiling strategy will be elaborated in Section 3.1 and the resulting prediction routines will be described in Section 3.2.

On top of performance, we also need to be able to predict the impact of our decisions on system power. For this purpose, we have trained a linear regression model that estimates the power costs of the predicted performance metrics. This model only involves platform-specific parameters and only has to be trained once for a given system. The power model is described in Section 3.3.

\subsection{Application Profiling}

As explained above, to guide the prediction of performance metrics under changing circumstances, we perform offline application profiling. Since this has to be done in advance, we cannot assume knowledge of the application mixes that will appear at runtime, thus we restrict ourselves to standalone profiling of each one application, contrary to works such as Reference [1], which propose model training for each mix separately. Furthermore, we note that the number of applications that can appear on a system during its lifetime is unpredictable. Thus, it is important that offline profiling for each one remains scalable, both in terms of time needed to complete and in terms of memory required to store the results that are used at runtime.

3.1.1 Performance Profiling at a Set Frequency. To find out how an application performs under different levels of system workload, we run multiple instances of it concurrently, varying the number of these instances from one up to the total number of cores in the system minus one. For each number of instances, we use three different policies for placing them to cores:

(1) Use the biggest available core.

(2) Use the smallest available core.

(3) Use the type that currently has more available cores (resolving ties in favor of the biggest).

For instance, when we run six instances on a system with four big and four little cores, they will be placed in the following ways by each of the three policies:

(1) Four big and two little cores.

(2) Two big and four little cores.

(3) Three big and three little cores.

The outcome of these runs is the set of initial states, i.e., the circles on the left side axis of Figure 2 for each of the performance metrics used in our prediction.

Additionally, for each of the initial states, we perform one more run for each type of core in the system, with one extra instance spawning on such a core, if possible. For example, after running the initial state of three big and three little cores, we also profile the following two states:

(1) Three big and four little cores $(+1 \mathrm{~L})$.

(2) Four big and three little cores $(+1 \mathrm{~B})$.

These runs correspond to final states, i.e., the squares on the right side axis of Figure 2. For each initial and final state, we store the values of the four performance metrics.

The above application profiling process is performed at a single, intermediate frequency $\left(f_{c}\right)$ of each cluster. We defined $f_{c}$ to be $1.4 \mathrm{GHz}$ for the big cluster and $1.0 \mathrm{GHz}$ for the little cluster. However, as described earlier in this section, the performance of each application is also affected differently by frequency. It would be possible to repeat the process in every frequency, but this would multiply both the time needed for profiling and the size of the resulting performance char- 
acterization file by a factor equal to the number of available frequency levels. Thus, we deal with the impact of frequency in an orthogonal manner, explained in the following subsection.

3.1.2 Frequency Scaling. The effect of frequency on performance depends on the memory- or compute-boundedness of the application. For an entirely compute-bound application, Instructions per Cycle (IPC) are the same at every frequency, resulting to IPS scaling linearly. In simpler terms, an entirely compute-bound routine that takes $1.0 \mathrm{~s}$ to complete at $500 \mathrm{MHz}$, would take $0.5 \mathrm{~s}$ at 1.0GHz. For a more memory-bound application, the effect of DVFS is diminished, meaning that IPC is lower at higher frequencies and the effect on IPS is sublinear. A memory-bound routine that takes $1.0 \mathrm{~s}$ to complete at $500 \mathrm{MHz}$, would take less than 1.0 but more than $0.5 \mathrm{~s}$ at $1.0 \mathrm{GHz}$, as doubling the frequency would only speed up computations, not memory accesses. To trace the effect of frequency on each application, we run a single instance of it on each core type, at every available frequency level and compute a scaling factor $(S F)$, which represents the average IPC degradation for a frequency increase of $100 \mathrm{MHz}$. Thus, in the general case, $S F \leq 1.0$ and for an entirely compute-bound application, $S F=1.0$. We obtain one scaling factor for each core type. At runtime, the scaling of IPC is converted to IPS. More specifically, the estimated performance $I P S_{\text {new }}$ of an application when changing the frequency from $f_{1}$ to $f_{2}$ is

$$
I P S_{\text {new }}=I P S_{\text {old }} *\left(f_{2} / f_{1}\right) * S F^{\alpha},
$$

where $f_{2} / f_{1}$ is the speedup (or slowdown) of computational parts due to the frequency change and $S F^{a}$ is the part of this speedup (or slowdown) that is mitigated by memory accesses, with

$$
\alpha=\left(f_{2}-f_{1}\right) / 100 \mathrm{MHz}
$$

being the number of $100 \mathrm{MHz}$ steps required to go from $f_{1}$ to $f_{2}$. Furthermore, for standalone execution of an application, the rest of the performance metrics (L1aps, LLCaps, DRAMaps) scale in the same manner as IPS, as the data access pattern remains the same across different frequencies, thus the amount of instructions and memory accesses is the same.

To profile one application on a generic board with $n$ cores, $l$ core types, and $r$ frequency levels for each core type, we need a maximum of $n * 3 * l+l * r$ runs, as for each of the $n$ instance counts we use three different placement policies to create the initial states and need $l$ extra runs to profile the final states of each initial state. On top of these, we need $r$ runs to determine the scaling factors of the application on each of the $l$ core types. While the number of system cores $n$ can be expected to increase in the future, the frequency levels $r$ and the different core types $l$ are not expected to increase as radically. Thus, the asymptotic complexity of our application profiling would be $O(n)$, considering constant $l$ and $r$. This is a loose upper bound, because many of the configurations occur more than once but only have to be profiled once; in other words, there is overlap between the three placement policies and between initial and final states. In total, on our available board of $n=8$ cores, $l=2$ core types and using $r=11$ different frequency levels on each core type, the loose upper bound is 70 , but because of overlap, we need to run each application in only 46 different configurations. As an indicative comparison to existing approaches, the characterization methodology proposed in Reference [9], needs to run each application in 128 configurations on the same board we use and if the same methodology were to be applied on larger systems unchanged, the asymptotic complexity would be $O\left((n / m)^{m}\right)$, where $m$ is the number of clusters in the system.

In terms of memory requirements, our profiling file for one application consists of 24 entries for each of the four performance metrics and for each core type. Each entry being 4 bytes, this results to $24 * 4 * 2 * 4=768$ bytes. The scaling factors (one for each core type) require an extra 8 bytes, for a total of 776. For a generic system, the number of entries produced by workload profiling is linear with respect to the total number of runs, while frequency profiling produces just $l$ scaling factors, one for each type of core. Thus, the memory required is asymptotically $O(n)$. 


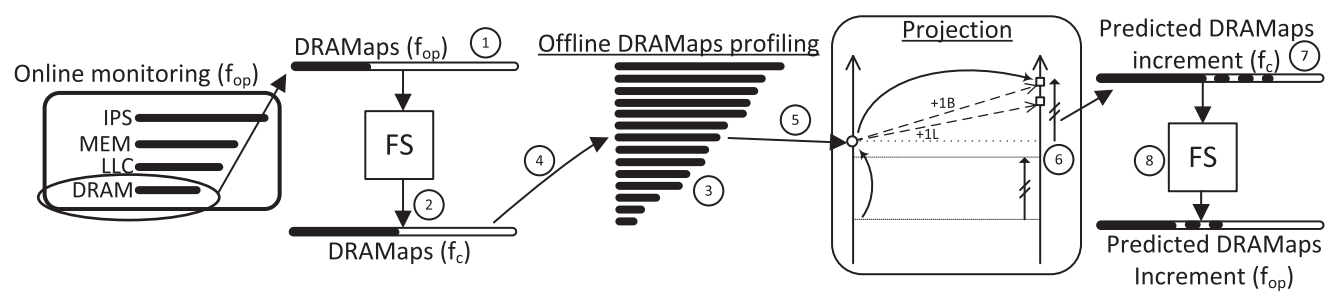

Fig. 3. Predicting performance of a newly spawned application using online projection. The current monitored value of DRAMaps at the operating frequency $f_{o p}$ (1) is scaled to the profiling frequency $f_{c}$ (2). The sorted profiled values for the new application's DRAMaps are fetched (3) and the one closest to the current is spotted (4) to be used as the "resembling state" of the projection (5). The resulting predicted DRAMaps (6) can be added to the system-wide aggregate (7). The result is scaled back to the current operating frequency, $f_{o p} 8$.

\subsection{Performance Prediction via Online Projection}

Our runtime manager makes decisions concerning the frequency level of each cluster and the placement of the current workload on available cores. Thus, it needs to accurately predict application performance as a result of the following two actions:

(1) Changing the frequency of a cluster.

(2) Placing a newly spawned application on a core.

To perform these predictions, the runtime manager combines application profiling data with online performance measurements of the applications currently running, as indicated in Figure 1. At all times, the current values of the four performance metrics are known for each application via online performance monitoring, thus also their aggregate values for each cluster and the whole system. The following explains how predictions are performed.

Changing frequency: One of the decisions that our runtime manager has to make, is to determine cluster frequencies. To do so, it has to be able to predict applications' performance at any candidate frequency. To this end, the runtime manager uses the scaling factors of all running applications and Equation (1), to predict how the value of each performance metric changes for each application. As explained in the previous subsection, while Equation (1) refers to IPS, the rest of the performance metrics scale identically.

To carry out the above process, the runtime manager needs to perform a constant amount of calculations for each application running on the examined cluster. Assuming that each core runs a maximum of one application, this results to an asymptotic complexity of $O(n / m)$, where $m$ is the number of clusters, thus $n / m$ is the number of cores per cluster. In case future systems maintain a similar count of cores per cluster, this cost can be considered constant.

Placing new application: When a new application spawns, the runtime has to choose a cluster with an idle core for it to execute. To guide this decision, it requires the ability to predict the effect of different placement options. This prediction is performed using our projection technique. Below, we outline the steps taken to predict all four performance metrics, while Figure 3 illustrates the process for the DRAMaps metric specifically:

(1) For each of the four performance metrics, the current value is determined, consulting the latest online monitoring results (1). These values, which are being monitored at the current operating frequency $f_{o p}$, are then scaled to the performance profiling frequency $f_{c}$, using Equation (1) for each application currently running (2). 
(2) For each of the four performance metrics, their profiled values corresponding to the starting states are fetched. These values are stored sorted (3, to find, in logarithmic time, the value closest to the current monitored value (4). This is the value of the resembling state, as it was defined in the opening part of Section 3 and Figure 2. As such, it is the initial profiled state that is used for the actual projection (5).

(3) Next, the runtime looks for the appropriate profiled final state, that is, the final state that corresponds to one extra instance of the profiled application on a core of the same type as the candidate cluster. This is the projected state of Figure 2. The difference between the values of the performance metric in the projected and resembling states (6) is the estimation for this performance metric, if the application is placed on the candidate cluster. This is also how much the system-wide aggregate value for this performance metric is expected to increase 7 .

(4) As this value was estimated with respect to the profiling frequency $f_{c}$, it is scaled back to the current cluster frequency, using Equation (1) 8.

The bottleneck of the projection process is finding the resembling value of each metric among the $O(n)$ sorted offline profiling values. The complexity of doing so is $O(\log n)$.

Predictions involving multiple actions: Occasionally, the runtime manager has to predict the impact of a decision involving multiple incremental steps of the two types explained above (frequency scaling, application placement). To do so, it takes the following steps:

(1) It performs the required prediction for the first incremental step.

(2) It updates the current system status with the predicted performance values in place of the currently monitored ones.

(3) It performs the required prediction for the next incremental step, based on the updated system status. The process is repeated for each step.

A good example of such a situation is the action of moving an application from one core to another. This is approached by the runtime manager as an application termination followed by a new application placement. The specific steps are as follows:

(1) The system status is updated to reflect the fact that the application in question will not execute on its current core anymore. More specifically, the values of the application's performance metrics are subtracted from the cluster- and system-wide aggregates.

(2) On this new system status, projection is performed as described above, to predict the application's performance on the candidate new core.

Another example is placing a newly spawned application, at the same time increasing the frequency of the chosen cluster (e.g., to satisfy a performance requirement of the new application). In a similar manner as above, the following steps are taken:

(1) Projection is performed to predict the performance metrics of the new application for placement on the candidate cluster. The system status is updated by adding the predicted performance metrics to the cluster- and system-wide aggregates, as shown in Figure 3.

(2) On this new system status, the frequency scaling routine is applied, to predict the impact of increasing the cluster frequency on all applications, including the one that just spawned and hasn't yet started executing.

As a general rule, the runtime system always uses online monitored values, when these exist. If such values are not available, then predicted values are used instead. 


\subsection{Power Prediction}

Besides predicting performance, a reliable way to predict system power is needed as well. Power prediction revolves around the same performance metrics, augmented with characterization of a specific system to obtain energy costs for instructions and memory accesses. Our model considers that the total power consumption of a cluster is the sum of its idle power, incremented by the contributions of aggregate IPS and accesses to the levels of the memory hierarchy, at a particular frequency. This is expressed by Equation (2), that estimates the total power for one cluster, $P_{c l}$ :

$$
P_{c l}=P_{\text {idle }}(f)+\gamma * I P S * P_{I}(f)+\text { L1aps } * P_{L 1}(f)+\text { LLCaps } * P_{L L C}(f)+\text { DRAMaps } * P_{\text {DRAM }}(f) .
$$

In Equation $(2), P_{\text {idle }}(f)$ is the power cost of a cluster currently not executing any application, at frequency $f$. IPS, L1aps, LLCaps, and DRAMaps are the values for the performance metrics, either measured by online monitoring or predicted as explained in Section 3.2. Furthermore, $P_{I}(f)$, $P_{L 1}(f), P_{L L C}(f)$, and $P_{D R A M}(f)$ are the energy costs of one occurrence of each performance metric, i.e., one instruction executed, one L1 access, and so on. The energy cost of one occurrence is equal to the power cost of one occurrence per second. As the performance metrics are measured in occurrences per second, each product corresponds to the power cost entailed by one performance metric, e.g., L1aps $* P_{L 1}(f)$ represents the total power cost of all L1 accesses at frequency $f$. Instructions are treated slightly differently, because the energy costs of different types of instructions can vary significantly, e.g., as demonstrated by Vasilakis et al., a floating point division on a big.LITTLE system has 4 to 8 times higher energy cost than an integer multiplication [26]. The $\gamma$ factor in the instructions term characterizes the instruction mix of an application and covers this variability. An application instruction mix and consequently its $\gamma$ factor is assumed to be constant and is determined offline for each application separately.

Characterizing a specific system in terms of power consumption consists of estimating all unknown values of Equation (2). To do so, we use the INA231 energy sensors of our available board [25], to measure total system power at specified intervals. Although there are finer-grain sensors on the board (i.e., power per cluster), we consider the limitation to total system power important, as more recent boards such as the ODROID XU4, often come without on-chip energy sensors because of cost concerns. Total system power is not dependent on finer-grain sensors, as it can be measured with alternative techniques, such as a sensor connected to the power outlet [8].

The easiest to determine unknown value is $P_{\text {idle }}$. We measure the power dissipation of the system while it executes no applications. The idle power value of each cluster can be estimated by fixing the frequencies of all other clusters to the lowest physically possible value, and varying the frequency of the examined cluster in steps of $100 \mathrm{MHz}$. This is how we determine the values of $P_{\text {idle }}$ on our available board, for each cluster and each frequency level.

To determine the values of the coefficients $P_{I}(f), P_{L 1}(f), P_{L L C}(f)$, and $P_{D R A M}(f)$, we perform linear regression using measured data obtained from a mix of synthetic and NAS benchmarks [15], covering a set of diverse situations (i.e., compute- and memory-bound, integer, and floating point arithmetic). As this is a step that needs to be performed only once, for a given platform, we repeat the process for each frequency of each cluster. The output of this stage is the "Platform Characterization" file in Figure 1, consisting of five floating point numbers (20 bytes) for each frequency of each cluster type.

As the training of this model only estimates platform-specific coefficients, it can be used with any application mix without re-training. Equation (2) can be used at runtime combined with performance prediction, to estimate the power impact of various actions (application placement, frequency scaling) or events (application termination). 


\section{THE HEURISTICS}

The prediction model described in Section 3 allows our runtime manager to have an estimation for the impact of possible decisions on power and performance. Still, it is infeasible to evaluate every possible choice at runtime, to apply the best one. This section describes the runtime heuristics we have developed to shorten this search. The organization of our system is shown in Figure 1. The currently selected heuristic is triggered by an event, that is a new application spawning or a running application termination. The heuristic selects a small number of candidate solutions to evaluate, by estimating their efficiency using the prediction model. As explained in detail in Section 3, the model combines platform characterization and application profiling information obtained offline, with runtime performance measurements obtained from the system online, to estimate the performance and power outcome of changing a cluster's frequency and of placing a new application. Thus, all presented algorithms have available the following two routines:

- frequency_predict $(c, f)$ : Predicts the outcome of setting the frequency of cluster $c$ to $f$,

- placement_predict $(a, i)$ : Predicts the outcome of placing application $a$ on core $i$.

To predict the efficiency of a new configuration, the runtime manager has to define a sequence of steps of the above two types that lead from the current system configuration, to the new one.

As analyzed in Section 3.2, these routines have a time cost of $O(n / m)$ and $O(\log n)$, respectively. In the following analysis, we consider the number of available frequency levels per cluster, $r$, and the number of different core types, $l$, as constants with respect to the total core count $n$.

We propose three different heuristics. They differ in the manner in which they choose candidate solutions to evaluate. A more flexible algorithm can search a larger part of the solution space and thus has a higher probability to find a more efficient solution. However, its execution time is longer, and it is possible that it scales less well when the system size increases. In Section 5, the advantages and drawbacks of each algorithm are demonstrated through experimental results.

\subsection{Heuristic 1}

The first heuristic we suggest is the simplest and less flexible of the three. It is described by the pseudocode of Algorithm 1. The algorithm starts by cycling through the system clusters (line 6). For each cluster, it checks whether or not there is an available core (line 8). If so, then it predicts the efficiency of the system if the application is placed on this free core (line 9). The cluster that yields the best predicted efficiency and the first available core in this cluster are chosen.

Subsequently, the system status is updated and the algorithm goes on to select a frequency for the chosen cluster. To do so, it calls routine choose_frequency(), shown in Algorithm 0. This routine performs a logarithmic number of steps (line 7). In each step, it predicts system efficiency in two extreme frequency levels, "high" and "low" (lines 8 and 9), and then readjusts them depending on which one produced the best efficiency prediction (line 13 or 17). In this manner, it narrows downs the candidate frequency range to half the size in each step, until one frequency is chosen.

The process of choosing a cluster consists of $m$ steps, each of which performs projection, which $\operatorname{costs} \log n$ time. The process of choosing a frequency consists of a constant number of calls to frequency_predict(), thus costs $n / m$ time. The resulting asymptotic complexity is $O(m * \log n+n / m)$. The scaling of the number of clusters $m$ defines which of the two terms of this sum is dominant. If the number of cores per cluster of bigger systems is the same as today's systems (e.g., four cores per cluster), then $m$ will scale linearly with $n$, making $n / m$ constant and the complexity of Heuristic $1 O(m * \log n)$. In the other extreme case, the number of clusters $m$ will remain constant and the number of cores per cluster $n / m$ will scale linearly with $n$, resulting to a complexity of $O(n)$. 

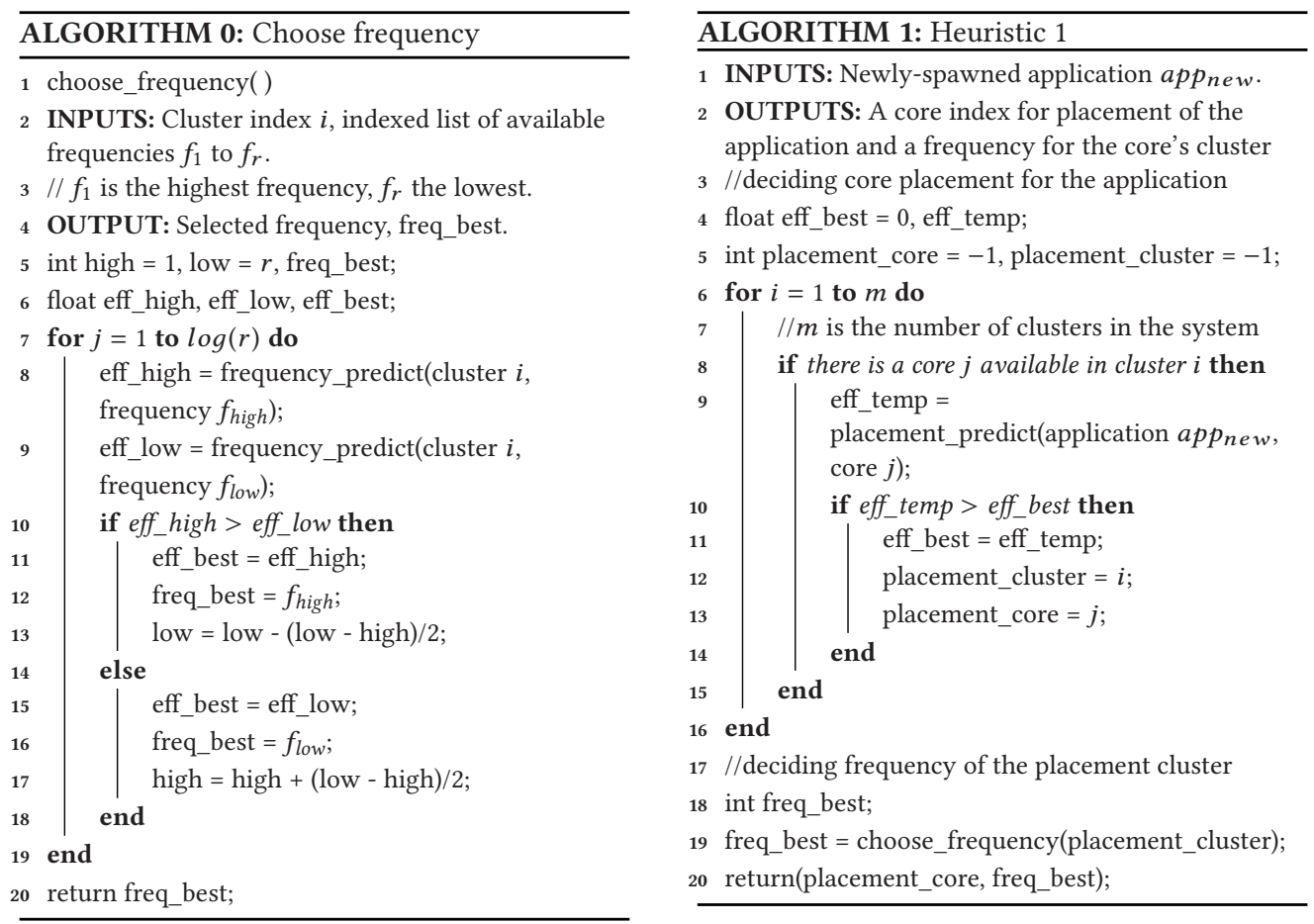

Note that the pseudocode describes the way Heuristic 1 deals with a new application spawning. All heuristics are also called whenever an application finishes execution. In this case, the part that determines the placement of the new application is skipped (i.e., lines 5-16 of Heuristic 1).

\subsection{Heuristic 2}

The second heuristic, described in Algorithm 2, is similar to the first, with the extra feature of estimating the impact of the newly-spawned application on the performance of other applications already executing on the chosen cluster. As applications share common resources in the system, i.e., the memory hierarchy, re-estimating the performance of already existing applications is expected to yield more accurate results. This additional feature can be spotted in lines $9-11$. Here, the algorithm cycles through all cores of the currently examined cluster. For each core that executes an application, it performs a projection to predict its performance in the new, more contested state and overwrites current monitoring data of these applications with the predicted values. Otherwise, just like Heuristic 1, this algorithm chooses the cluster and core with the highest predicted system efficiency and then chooses a frequency for this cluster. Just like Heuristic 1, if the calling event was an application termination, lines $4-18$, corresponding to the placement decision, are skipped. Heuristic 2 performs $m$ sets of up to $n / m$ projections, resulting to a time cost of $O(n * \log n)$.

\subsection{Heuristic 3}

The third heuristic, described by Algorithm 3, is the most complex of the three, as it is the only one able to take corrective action, by moving already placed applications between cores. As such, it is the only heuristic that allows for revisiting previous runtime decisions. Initial decisions are made using profiled application information. Revisiting an initial decision allows the use of real, monitored performance data, in place of less accurate predicted performance, about an application. 

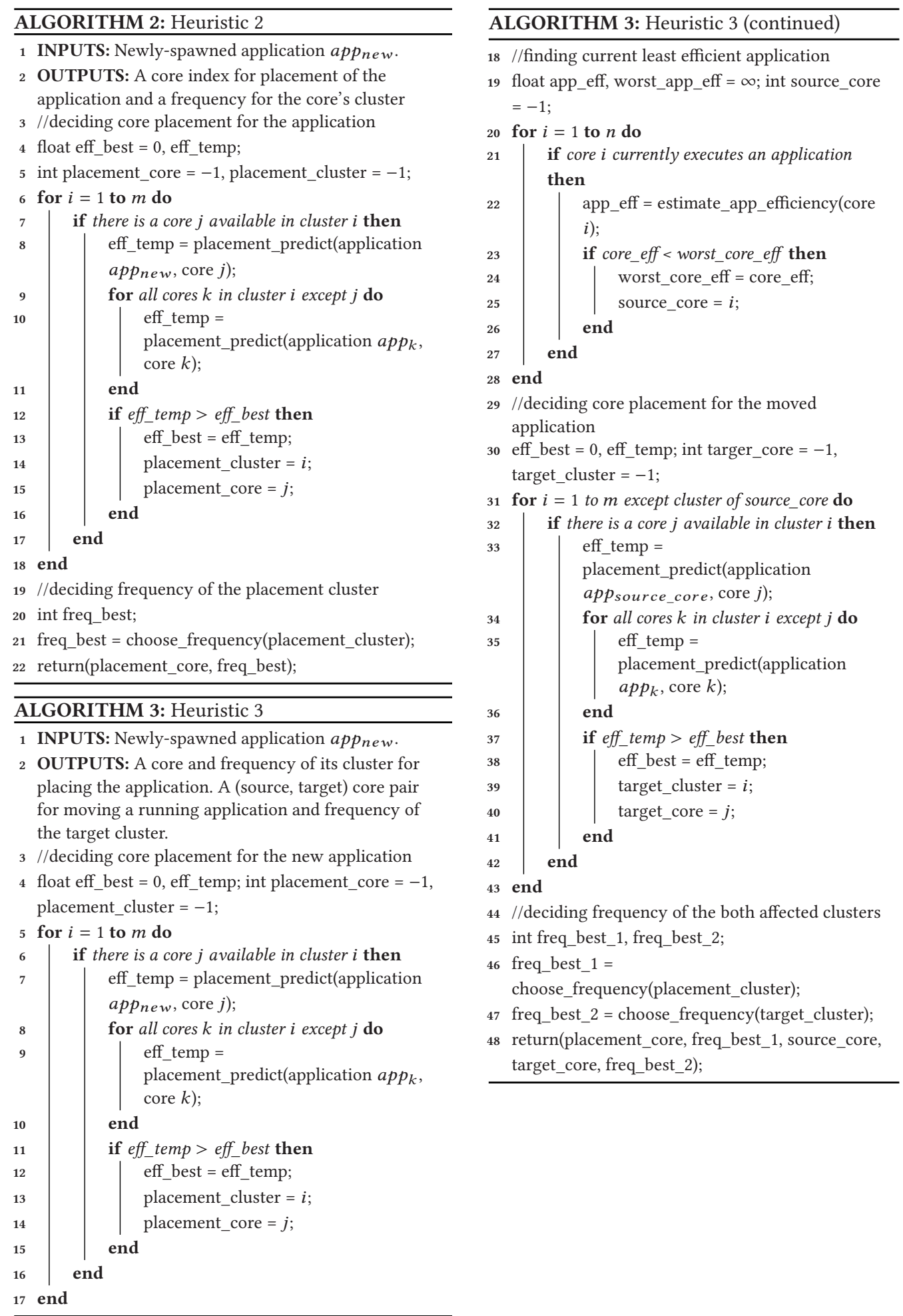
Heuristic 3 starts by choosing a placement for the new application in the same manner as Heuristic 2 (lines 5-17). Subsequently, it chooses a currently running application to be moved to a different core. To do so, it evaluates the efficiency of each running application, based on its actual online monitored performance and its estimated power, calculated with Equation (2) (lines 18-28). Next, it chooses a target core for this application on a different cluster aiming to maximize efficiency (lines 29-43). Last, the best frequencies are chosen, both for the placement cluster of the new application as well as for the target cluster of the moved application (lines 55-59). Note that in case the algorithm was called as a result of an application termination, only the new application placement part is skipped (lines 5-17). Moving applications is still possible.

The three main for-loops of Heuristic 3 have an asymptotic complexity of $O(n * \log n), O(n)$ and $O(n * \log n)$, respectively. Thus, its overall complexity is $O(n * \log n)$, but in absolute terms it is expected to take longer than Heuristic 2, despite having the same complexity.

\subsection{Dealing with Performance Requirements}

Concluding our heuristics' description, we present some rules concerning specific cases, mainly dealing with applications that have a performance requirement and need priority treatment. The notes of this subsection do not change the complexity of any algorithms and are left out of the initial description to reduce clutter.

First, any system configuration that does not respect performance requirements of applications is considered invalid and cannot be chosen. This is implemented by having the various routines return an efficiency value of 0 if the IPS prediction of an application is below the required. To exemplify, in line 9 of Algorithm 1, the routine placement_predict() is called, which involves predicting the IPS of application $a p p_{n e w}$. If this predicted IPS is lower than the performance requirement of $a p p_{n e w}$, then the routine returns an efficiency value of 0 , ruling out this placement option. Another example is routine frequency_predict() in line 9 of Algorithm 0. If the low frequency does not satisfy some application's performance requirement, then it is considered to have an efficiency of 0 . Note that the above means that an application not currently fulfilling its requirement is a candidate to be moved to a bigger core by Heuristic 3 , as its estimated efficiency is also 0 .

On a related note, all algorithms' decisions are partially based on predictions, which cannot be perfect. When performance requirements exist, this inaccuracy has to be outweighed by a degree of pessimism in the decision. To achieve this, a slack value is defined for each algorithm. Whenever the performance of an application with a requirement is predicted, the algorithm attempts to fulfill a tighter requirement IPS_tight, instead of the real one, IPS_min, such that

$$
\text { IPS_tight }=I P S \_ \text {min } *(1+\text { slack }) \text {. }
$$

The choice of slack values in our experiments is guided by the average and worst-case inaccuracy of our performance model, presented in Section 5.1.

Furthermore, applications with a requirement are given priority over other applications when it comes to core placements. When a constrained application is placed, all cores executing a nonconstrained application are considered potentially free (of course, an actually free core on the same cluster is always preferred). Thus, checks such as line 8 of Algorithm 1, take this into consideration. The application that is bumped out of a core in this case is placed on the most similar available core.

Last, an application that cannot be placed, because there is no available core in the system, is put on hold until a core becomes available. In accordance with the rule stated in the previous paragraph, this is not true if the application has a performance requirement-in which case, another application is paused. 
Table 2. The Applications Used for Calibration of the Power Prediction Model (Left) and for Evaluation (Right)

\begin{tabular}{|l|l||l|l|}
\hline \multicolumn{2}{|c||}{ Calibration Applications } & \multicolumn{2}{c|}{ Evaluation Applications } \\
\hline FT - class A (NAS) & FP arithmetic (synthetic) & CG - class A (NAS) & Susan large, all (MiBench) \\
\hline BT - class A (NAS) & Integer arithmetic (synthetic) & IS - class A (NAS) & Linpack_2000 sp (Miscellaneous) \\
\hline EP - class A (NAS) & \multirow{3}{*}{$\begin{array}{l}\text { Vector with random column } \\
\text { multiplication (synthetic) }\end{array}$} & SP - class A (NAS) & Matmul_512 dp (Miscellaneous) \\
MG - class A, 2 different phases (NAS large (MiBench) & Whetstone dp (Miscellaneous) \\
LU - class A, 2 different phases (NAS) & & Bitcount large (MiBench) & NQueens_15 (Miscellaneous) \\
\hline & & Qsort huge (MiBench) & \\
\hline
\end{tabular}

\section{EVALUATION}

In this section, we present experimental results evaluating our approach. Our experiments were performed on an ODROID XU3 board [20], which is built around an Exynos 5422 Processor, consisting of a cluster of four "LITTLE" Cortex-A7 cores and a cluster of four "big" Cortex-A15 cores. Each core has a private L1 data cache of $32 \mathrm{~KB}$, while L2 is shared per cluster and is $512 \mathrm{~KB}$ for the little cluster and 2MB for the big. The two clusters share a 2GB LPDDR3 RAM main memory. The block diagram of the Exynos chip is shown at the upper part of Figure 1, labelled "Hardware." We have determined the frequency range of the little cluster to be between $500 \mathrm{MHz}$ and $1.4 \mathrm{GHz}$ and that of the big cluster between $800 \mathrm{MHz}$ and $1.8 \mathrm{GHz}$-both in steps of $100 \mathrm{MHz}$. We have observed that scaling the frequency below these points does not reduce the voltage, offering diminishing power benefits. Other related works have limited the frequency range similarly [7].

We have used two sets of applications in this work. The first consists of NAS [15] and synthetic benchmarks listed at the left of Table 2 and was used for calibrating the power estimation model expressed by Equation (2). The second set consists of NAS [15], the automotive MiBench suite [10] and miscellaneous benchmarks listed at the right of Table 2 and was used to evaluate our proposed approach. We tried to have a balanced mix of compute- and memory-bound applications in both sets, to achieve both a good model calibration and a fair evaluation. When it came to choosing which NAS benchmarks would be part of our evaluation suite, we selected the ones the execution time of which was of the same order of magnitude as the rest of the benchmarks (less than a minute), to keep experimentation time within practical boundaries (around ten minutes per run).

The application spawning for the experiments described in Sections 5.1 and 5.2 was done by an event generator tool, that generates exponentially distributed application spawn events based on the following parameters for each application:

- A spawn rate, determining how often the application spawns. Multiple concurrent instances of an application are allowed to exist, so we run multiple random simulations of the exponential distribution in parallel, some of which spawn an event in the allotted time.

- A workload range that determines how long the application runs, defined by a minimum and maximum number of iterations that have to complete before terminating. For each spawn event, a value from this range is chosen in a uniform random manner. The runtime manager is not aware of this number, as completion times are supposed to be unpredictable.

- A probability of the application having a performance requirement, and a range of possible performance requirements, expressed in IPS. The event generator first decides whether or not the spawned application has a performance requirement and if so, it chooses a performance value from the available range in a uniform random manner.

The runtime manager runs on a little core, unless otherwise noted. 
Table 3. Exhaustive Evaluation of the Prediction Model, Our Heuristic, and the Strategy as a Whole

\begin{tabular}{|c|c|c|c|c|c|c|c|c|}
\hline & & & \multicolumn{3}{|c|}{ Model Misprediction } & \multicolumn{3}{|c|}{ Solution Efficiency vs. Optimal } \\
\hline \# & $\begin{array}{l}\text { Core } \\
\text { Util. }\end{array}$ & Application & $\begin{array}{l}\text { Performance } \\
\text { Mean (std dev) }\end{array}$ & $\begin{array}{c}\text { Power } \\
\text { Mean (std dev) }\end{array}$ & $\begin{array}{c}\text { Efficiency } \\
\text { Mean (std dev) }\end{array}$ & $\begin{array}{c}\text { Model + } \\
\text { Exhaustive }\end{array}$ & $\begin{array}{c}\text { Oracle Model } \\
+ \text { Heuristic }\end{array}$ & $\begin{array}{l}\text { Model + } \\
\text { Heuristic }\end{array}$ \\
\hline 1 & Low & Basicmath & $1.52 \%(1.34 \%)$ & $8.87 \%(5.24 \%)$ & $9.40 \%(5.99 \%)$ & $4.0 \%$ & $4.3 \%$ & $5.1 \%$ \\
\hline 2 & Medium & Basicmath & $0.95 \%(0.72 \%)$ & $8.58 \%(5.59 \%)$ & $9.18 \%(6.61 \%)$ & $1.3 \%$ & $1.2 \%$ & $1.3 \%$ \\
\hline 3 & High & Basicmath & $0.76 \%(0.60 \%)$ & $7.11 \%(4.73 \%)$ & $7.50 \%(5.37 \%)$ & $2.2 \%$ & $1.4 \%$ & $1.6 \%$ \\
\hline 4 & Low & IS & $1.85 \%(1.37 \%)$ & $2.85 \%(1.60 \%)$ & $3.80 \%(2.40 \%)$ & $3.0 \%$ & $4.5 \%$ & $5.5 \%$ \\
\hline 5 & Medium & IS & $3.20 \%(2.38 \%)$ & $3.28 \%(2.40 \%)$ & $3.81 \%(2.86 \%)$ & $8.0 \%$ & $0.5 \%$ & $3.9 \%$ \\
\hline 6 & High & IS & $4.68 \%(2.87 \%)$ & $4.24 \%(3.10 \%)$ & $3.18 \%(2.85 \%)$ & $0.2 \%$ & $1.9 \%$ & $0.2 \%$ \\
\hline 7 & Low & Matmul & $4.52 \%(4.17 \%)$ & $6.90 \%(6.52 \%)$ & $5.62 \%(5.13 \%)$ & $0.6 \%$ & $5.5 \%$ & $3.1 \%$ \\
\hline 8 & Medium & Matmul & $3.27 \%(2.93 \%)$ & $7.12 \%(6.03 \%)$ & $6.37 \%(5.08 \%)$ & $6.7 \%$ & $3.7 \%$ & $4.6 \%$ \\
\hline 9 & High & Matmul & $1.92 \%(1.82 \%)$ & $5.64 \%(4.15 \%)$ & $5.12 \%(3.98 \%)$ & $1.4 \%$ & $2.1 \%$ & $1.4 \%$ \\
\hline \multicolumn{3}{|c|}{ Average (min, $\max )$} & $2.52 \%(0.76 \%, 4.68 \%)$ & $6.07 \%(2.85 \%, 8.87 \%)$ & $6.00 \%(3.18 \%, 9.4 \%)$ & $3 \%(0.2 \%, 8 \%)$ & $2.5 \%(0.5 \%, 5 \%)$ & $3 \%(0.2 \%, 5.5 \%)$ \\
\hline
\end{tabular}

\subsection{Exhaustive Evaluation for Single Events}

In this section, we perform exhaustive evaluation of the prediction model and the heuristics, by comparing to an oracle model and an exhaustive search algorithm, respectively. We base this evaluation on nine representative application spawn events and examine in depth our model's and heuristic's response to them. The nine events are created as follows:

(1) With our event generation tool, we construct three starting states for the system, corresponding to low (25\%), medium (50\%), and high (75\%) core utilization.

(2) We select three of the evaluation applications: Basicmath being completely computebound, Matmul as the most memory-bound (using a large dataset) and IS as one of the intermediate applications in terms of memory boundedness.

(3) We spawn each of the three applications on each of the three starting system states.

For each event, we enumerate all possible configurations, in other words all combinations of new application placement, frequency of little cluster and frequency of big cluster. We use our model to predict performance, power and energy efficiency (in terms of IPS/Watt) for each possible decision (system configuration). Then, we apply all possible decisions one by one and measure the actual performance, power and efficiency. The left part of Table 3, titled "Model Misprediction," shows, for each event, the average inaccuracy of our model in predicting these three figures, across all possible decisions.

We observe that both parts of our model perform satisfactorily, as the average error is always less than $10 \%$. Performance prediction is more accurate (2.5\% average error) than power $(6.1 \%$ average error). In some cases performance and power mispredictions add up, resulting to a higher efficiency error (e.g., event \#1), while in other cases the opposite happens (e.g., event \#6). Furthermore, the performance of the compute-bound application Basicmath is easier to predict (maximum error $1.52 \%$ ), as it does not depend much on contention for shared resources, thus it is more straightforward to infer from offline data. However, the performance misprediction for IS and Matmul, which are harder to predict, is not much worse (maximum error $4.68 \%$ ).

Subsequently, for each event, we compare the efficiency of the following four runtime managers:

(1) An exhaustive oracle predictor that uses the actual measurements to exhaustively search and select the most efficient configuration. Since it always makes the optimal choice, this manager is used as a baseline. 
Table 4. The Application Mixes Used for Energy Efficiency Evaluation of the Proposed Heuristics

\begin{tabular}{|l|l|l|}
\hline Mix \# & \multicolumn{1}{|c|}{ Description } & \multicolumn{1}{c|}{ Applications } \\
\hline \hline 1 & NAS Benchmarks & CG, IS, SP \\
\hline 2 & MiBench Benchmarks & Basicmath, Bitcount, Qsort, Susan \\
\hline 3 & Miscellaneous Benchmarks & Linpack, Matmul, Whetstone, NQueens \\
\hline 4 & Compute-bound & Basicmath, Bitcount, Whetstone, NQueens \\
\hline 5 & Memory-bound & Matmul, CG, SP \\
\hline 6 & Uniform balanced & Susan, Linpack, IS \\
\hline 7 & Non-uniform balanced 1 & Basicmath, SP, Linpack, Susan \\
\hline 8 & Non-uniform balanced 2 & Bitcount, CG, Linpack, IS \\
\hline
\end{tabular}

(2) Our prediction model, exhaustively evaluating all possible decisions and applying the one it thinks best. This step evaluates our prediction strategy, independent of the heuristics' quality.

(3) Heuristic 1 guided by an oracle predictor. This step evaluates our basic heuristic independent of the quality of our prediction model.

(4) Heuristic 1 guided by our prediction model. This is our complete proposed approach.

The last two steps focus on only one heuristic, due to the complexity and duration of the required exhaustive experiments. However, the efficiency of the rest of our heuristics is comparatively evaluated in the next subsection. The right half of Table 3 shows the efficiency loss of using managers 2 ("Model + Exhaustive"), 3 ("Oracle Model + Heuristic"), and 4 ("Model + Heuristic"), compared to the optimal exhaustive oracle predictor.

When it comes to accuracy of the final decision, we observe that the efficiency loss of exhaustively evaluating all possible configurations with the model (Model + Exhaustive), is 3\%, which is lower than the average misprediction of the model (6\%). This happens because misprediction is usually uniform among different configurations (i.e., either always optimistic or always pessimistic). Thus, despite the absolute error, the model consistently captures the efficiency trend, resulting to a configuration very close to the optimal one (same placement of the new application, very similar cluster frequencies). The only exception is event \#5. For all three IS scenarios, the placement decision (big or little core) makes less than $10 \%$ difference in the final efficiency. Thus, in one case the misprediction is enough for the model to choose the wrong core placement, resulting to $8.0 \%$ energy efficiency loss compared to the optimal. Furthermore, when applying Heuristic 1 guided by the oracle predictor, we get an average $2.5 \%$ energy efficiency loss compared to the optimal, proving that the heuristic searches the correct part of the solution space-the efficiency loss is due to small deviations from the optimal frequencies. This is only slightly increased to 3\%, when we use the actual model in place of the oracle, reinforcing our previous point of the model misprediction being absorbed when comparing different candidate configurations.

\subsection{Evaluation with Dynamic Scenarios}

In this section, we evaluate our approach as a whole, with an extensive set of unpredictable, dynamic multi-application scenarios. We combine the evaluation applications of Table 2 into eight application mixes, summarized in Table 4. Mixes 1 to 3 consist of applications coming from the same source (NAS, MiBench automotive and miscellaneous benchmarks). Note that mix 2 is also used in similar experiments of Reference [7]. Mixes 4 to 8 aim to create different combinations of compute- and memory-bound applications. We have sorted all applications in order of decreasing 
scaling factor (SF)-the lower the SF, the more memory-bound the application is. Based on this classification, we include the most compute-intensive applications in mix 4, the most memorybound ones in mix 5 and the intermediate ones in mix 6 (hence called "Uniform Balanced"). Mixes 7 and 8 are also balanced, but they include applications from both extremes, instead of just the intermediate group.

For each of the eight mixes, we use our event generator to create 20 sequences of application spawns in random, exponentially distributed moments in time. Among the 20 event sequences, we vary the applications' spawn rates in such a way that the peak system utilization is expected to be $25 \%$ in the first sequence, to $75 \%$ in the last. We allow applications to spawn during the first two minutes of each run. Subsequently, we carry on the run until all applications have terminated. We evaluate the efficiency of the run in terms of average IPS/Watt, based on the total instructions executed across all applications, the total time that the experiment took until all applications' completion and the average power during this time.

In the experiments of this section, we evaluate all three of our heuristics. On top of this, we also evaluate an alternative version of Heuristic 3 (called "Alt. Heuristic 3"): in this version, in addition to application spawnings and terminations, the algorithm is also called whenever a running application completes one iteration and updates its online monitoring data. In this manner, we give Heuristic 3 the chance to take corrective action in more regular intervals. We compare our heuristics both with each other and with existing Linux governors, namely the powersave, interactive and ondemand governors, as is common in related works [1,9]. For all experiments, we keep the default idle governor active, allowing it to put cores to idle states, when they do not execute an application [16]. Generally speaking, the powersave governor is more energy-efficient, as it chooses the lowest available frequency, which tends to be the most efficient, especially in the range we have chosen to allow. However, for the same reason, the powersave governor cannot fulfill performance requirements of applications, contrary to both interactive and ondemand, that prefer higher frequencies. To provide a complete and fair evaluation versus all available governors, we perform the following two separate sets of experiments:

(1) Ignoring the applications' performance requirements. This set of experiments evaluates our approach mainly versus the powersave governor, in the simpler scenario in which performance considerations do not exist and the only goal is maximizing energy efficiency (IPS/Watt).

(2) Considering the applications' performance requirements. To fulfill the requirements, our algorithms have to choose, on average, higher frequencies than in set (1). This usually entails an efficiency penalty, making it hard to compete versus the powersave governor. This set of experiments aims to demonstrate how our approach is able to fulfill the performance requirements, while at the same time maintaining an efficiency advantage versus the governors that are able to achieve this (interactive and ondemand).

The efficiency results for set (1) are illustrated in Figure 4(a) and for set (2) in Figure 4(b). For each application mix and runtime manager, the efficiency shown is the mean across the 20 runs, after each run is normalized to the value for the powersave governor. Figure 4(b) also shows the average performance degradation with respect to individual applications' performance requirements.

We observe the following in Figures 4(a) and 4(b):

Comparison to governors: We observe that, without performance requirements, our approach matches the efficiency of the powersave governor, being up to $3 \%$ better on average (using Heuristic 3) and is twice as efficient compared to the other governors. In this experiment without performance requirements, being on par with the powersave governor is especially important. When performance requirements are introduced, our approach can satisfy all of them, with Heuristic 


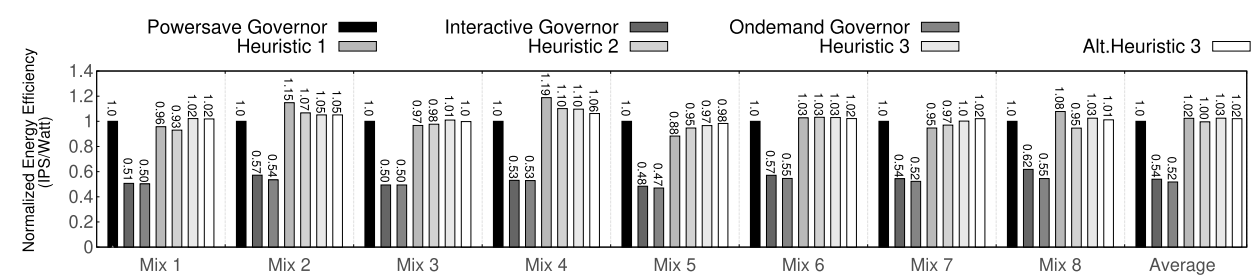

(a) Without Performance Requirements

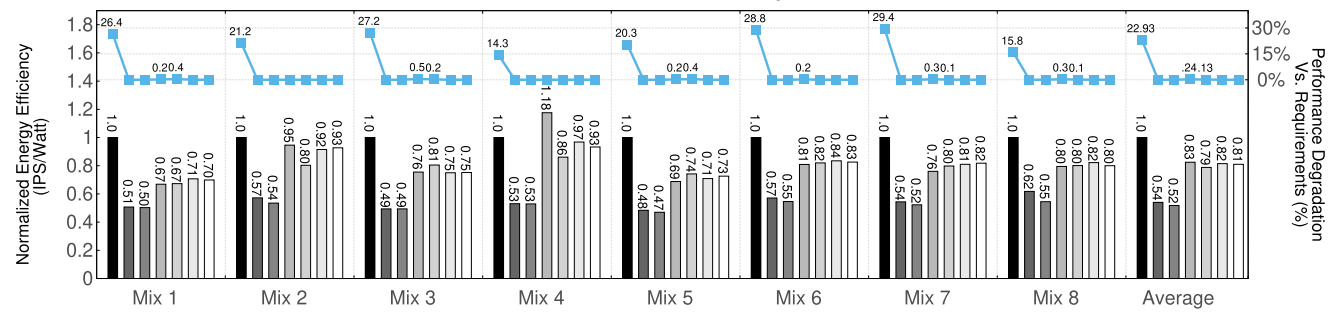

(b) With Performance Requirements

Fig. 4. Energy efficiency in terms of IPS/Watt, for all governors, heuristics, and application mixes, without (a) and with (b) performance requirements. All values are normalized to the efficiency of the powersave governor. When considering performance requirements, the average performance degradation compared to the requirement is also shown (scale on the right-side vertical axis).

3. Even Heuristic 1 satisfies almost all requirements, resulting to an average degradation versus target performance requirement of $0.24 \%$. Dealing with requirements comes at an expense of $17 \%$ to $21 \%$ of efficiency versus the powersave governor, which, however, does not fulfill requirements (it is on average $23 \%$ off the performance target). Additionally, Heuristic 3 achieves $52 \%$ and $58 \%$ better efficiency than the interactive and ondemand governor, respectively, while satisfying the same requirements.

Comparison among the heuristics: The relative energy efficiency of the heuristics depends on the scenario (application mix and presence or absence of performance requirements). Without performance requirements, Heuristic 1 is better with mixes 2 and 4 that consist mostly of computeintensive applications. Predictions for these applications are more straightforward, as there is no contention in the levels of the memory hierarchy. Thus, the simpler approach of Heuristic 1 is sufficient to detect an efficient configuration, while heuristics 2 and 3 gain nothing by their detailed prediction and/or corrective action capability. In fact, these extra steps are potential sources of misprediction, which sometimes hurts decision efficiency. The opposite trend is observed for mixes 1 and 5, consisting mostly of memory-bound applications. Performance of these applications varies more with respect to the current system status, thus the deeper prediction scheme of Heuristics 2 and 3 and the corrective action potential of Heuristic 3 benefit efficiency. Balanced mixes (3, 6, 7 , and 8 ) show the three heuristics achieving very similar results. Still, Heuristic 3 is best in mixes 3 and 7 by $3.1 \%$ to $5.3 \%$ and is only $0.2 \%$ behind in mix 6 .

Introducing performance requirements also complicates predictions, as the runtime manager has to raise the frequency enough to satisfy the requirement but not more, in order not to penalize efficiency more than needed. As a result, Heuristic 1 is relatively worse in some of the mixes (1 and 8), while it also misses more performance requirements than the other heuristics. Its average efficiency is still as good as Heuristic 3, mainly because of the outlier value it scores for mix 4. Heuristic 3 is best in 4 out of the 8 mixes, while its ability for corrective action (prioritizing performance requirements when doing so) results in all requirements being satisfied. 
Comparing the original version of Heuristic 3 with its alternative version, which is invoked more frequently, we find that it does not benefit from increasing the chances for corrective action. The reason is that, in our current implementation, application migrations can only happen between two iterations of an application. If a migration is decided, then the iteration currently running, starts over on the new core. This generally penalizes Heuristic 3, but its importance is increased for the alternative version that performs more migrations. Occasionally, we notice the alternative version performing the same migrations as the original but sooner, resulting to incremental gains (mixes 5 and 7). As the original version eventually takes the same action, this benefit remains low. However, in most mixes, especially mix 4, the alternative version migrates applications more aggressively, penalizing overall efficiency because of the reason explained above.

Comparison to related works: Our results show improvement compared to related works in different ways.

The most recent approach that evaluates dynamic multi-application scenarios [7], unfortunately does not compare to the powersave governor. The most relevant comparison to this work is versus an allocator aiming to maximize energy efficiency (MAX-EE), which is described as "an optimal brute-force allocation for maximizing IPS/Watt." It has to be noted however, that this allocator occasionally achieves lower IPS/Watt than others, meaning that it cannot be optimal with respect to the reported result. It is, of course, possible that it is optimal in a short-term manner, with respect to individual decisions taken throughout a run-which does not guarantee optimal energy efficiency for the whole run. Additionally, for this experiment, the various allocators being compared are guided by an oracle predictor instead of the model proposed in the SPARTA paper. Furthermore, although SPARTA is evaluated both through simulations and through runs on an ODROID XU3 board, comparison with the MAX-EE allocator is only performed in the former. Thus, on the simulated ODROID XU3, the SPARTA allocator achieves around 3\% worse energy efficiency than the brute-force MAX-EE allocator, while our approach achieves 3\% better than the powersave governor. Additionally, the execution time of the SPARTA allocator [7] explodes when the system size exceeds 32 cores, which, as will be shown in Section 5.3, is not true for our approach.

It is also important to compare with very recent works that focus on a slightly different paradigm [1, 9], as they do not evaluate for dynamic, multi-application scenarios. DyPO [9], which improves the results of Reference [1], achieves 6\% efficiency improvement over the powersave governor, which is however allowed to select frequencies as low as $200 \mathrm{MHz}$. On the contrary, we limit the powersave governor to a minimum frequency of 500 and $800 \mathrm{MHz}$ on the little and big clusters, respectively, as for lower frequencies voltage is not further reduced, diminishing the power benefits. According to our experiments, the powersave governor is at least $19 \%$ more energy efficient with our setup. On this setup, our approach achieves 3\% improvement versus the powersave governor.

\subsection{Execution Time of the Heuristics}

In this section, we report our heuristics' execution time and how this scales with respect to system size. These results are summarized in Table 5. We scale the number of cores in the system from 8 (the size of our available board) to 128. For sizes more than eight cores, we measure execution time for the cluster size of our available board (four cores) as well as for double this size (eight cores).

Comparing between the algorithms, the execution time of Heuristic 1 grows slower: It takes 2.6 to 2.8 times more for a 128-core system than for an 8-core system, whereas for heuristics 2 and 3 these factors are 4.1 to 4.3 and 4.1 to 4.5 , respectively. Furthermore, the execution time of Heuristic 1 grows faster for systems with more clusters of fewer cores, than for systems with fewer clusters of more cores, which is expected due to its dependence on the number of clusters, $m$. Indeed, for 
Table 5. Execution Time of the Three Proposed Heuristics for Various System and Cluster Sizes

\begin{tabular}{|c|c|c|c|c|c|c|c|c|c|c|}
\hline & \#cores (n): & 8 & \multicolumn{2}{|c|}{16} & \multicolumn{2}{|c|}{32} & \multicolumn{2}{|c|}{64} & \multicolumn{2}{|c|}{128} \\
\hline & \#clusters (m): & 2 & 2 & 4 & 4 & 8 & 8 & 16 & 16 & 32 \\
\hline \multirow{2}{*}{$\begin{array}{l}\text { Heuristic } 1 \\
\mathrm{O}\left(\mathrm{m}^{*} \log \mathrm{n}\right)\end{array}$} & Big, $800 \mathrm{MHz}$ & $80 u s$ & 104us & 74us & 113us & 103us & 146us & 135us & 193us & 206us \\
\hline & Little, $500 \mathrm{MHz}$ & 170us & 240us & 197us & 281us & 248us & 338us & 330us & 436us & 475us \\
\hline \multirow{2}{*}{$\begin{array}{l}\text { Heuristic } 2 \\
\mathrm{O}\left(\mathrm{n}^{*} \log \mathrm{n}\right)\end{array}$} & Big, $800 \mathrm{MHz}$ & 95us & 144us & 135us & 170us & 172us & 241us & 253us & 383us & 411us \\
\hline & Little, $500 \mathrm{MHz}$ & 215us & 305us & 297us & 383us & 386us & 539us & 550us & 857 us & 887 us \\
\hline \multirow{2}{*}{$\begin{array}{l}\text { Heuristic } 3 \\
\mathrm{O}\left(\mathrm{n}^{*} \log \mathrm{n}\right)\end{array}$} & Big, $800 \mathrm{MHz}$ & 128us & 202us & 191us & 241us & 243us & 347 us & 356us & 567us & 576us \\
\hline & Little, $500 \mathrm{MHz}$ & 301us & 409us & 407 us & 547us & 552us & 761us & 772us & 1210us & 1229us \\
\hline
\end{tabular}

8-core clusters, Heuristic 1 takes 1.9 times longer on a 128-core system compared to a 16-core system, whereas for 4-core clusters this factor is 2.8 .

More importantly, all heuristics' execution time scales sublinearly with system size, as it never doubles when doubling $n$. This shows that the theoretical upper bounds discussed in Section 4 are pessimistic. This result proves that all algorithms can be used on systems many times larger than the one we have experimented on, without concerns about their response time because of their ability to perform partial search of the configuration space without sacrificing much efficiency. As an indication of how the configuration space grows with respect to system size, we have measured the execution time of a lightweight version of exhaustive search, which considers all options, but only for the new application placement and for the frequency of the selected cluster. We found the execution time of this algorithm to be at least double than our longest heuristic for the smallest system size. More importantly, it grows much faster than our heuristics when increasing the system size: when going from 8 to 128 cores, this limited exhaustive algorithm takes at least 16 more times to execute, compared to the 2.6 to 4.5 factors reported above for the heuristics. Furthermore, this limited exhaustive search, does not even consider the full configuration space. To do so, it would have to also consider all possible migrations of current applications, which would multiply execution time by a factor growing exponentially with the system size.

\section{DISCUSSION}

In this section, we list some limitations and alternatives of the proposed approach, both to clarify our contributions and to set some targets for future improvements.

As is evident by the presentation of our performance prediction strategy in Section 3, it is assumed that applications appearing at runtime have been profiled offline. The results of the offline profiling are used as a starting point for the projection-guided runtime prediction. Our approach is indeed partly based on this offline step, thus we focus on making it feasible, by imposing two constraints: (i) profiling of each application should be standalone, regardless of the possible application mixes that can appear at runtime, and (ii) it should consist of a linear (with respect to the target system size) number of runs. The above does not mean that our runtime manager is unable to handle unknown applications, just not as accurately as profiled ones. If an application is unknown in the most extreme sense (not even one profiling run on one core type was possible to carry out offline), then at runtime it would initially have to be placed on an arbitrary core and its online performance monitoring would subsequently have to be used for any future decisions regarding this application, much like is done in Reference [7].

On a related note, when profiling an application, we only use its own instances to create the initial states. A possible alternative of this stage would be to manually construct a number of custom initial states, using a set of applications with variable characteristics. In this manner, we would potentially create a broader spectrum of initial states, with varying contention for shared resources, 
increasing the chances for one of them to be closer to the actual current system state. This might improve the accuracy of projection in some cases. Our current approach has the following two interesting characteristics compared to the above alternative. First, using multiple instances of the same application makes profiling standalone and simpler as it does not require the use of other applications. Second, an application with limited demand for a particular type of resource (e.g., DRAM accesses per second) is expected to suffer limited impact by applications that heavily compete for this same resource. Thus, using some fixed applications to form the initial states stressing competition for particular resources may not necessarily improve projection accuracy.

A limitation, regarding mostly our experimental setup, is the assumption of at most one thread per core. As the ODROID XU3 offers one set of performance counters per core, it is challenging to monitor more than one thread running concurrently on the same core and keep the measurements independent and consistent. However, regardless of the current implementation, our approach in general is not limited in this respect. Allowing multiple threads per core would not change the complexity of the runtime heuristics, as the least busy core of each cluster (with respect to its utilization) would still be preferred for application placement. However, profiling would indeed have to be extended, running each application for up to $c * n$ instances, instead of $n, c$ being a constant representing the maximum number of threads per core expected in runtime situations. In this manner, the contention between threads for processor cycles would also be considered, when performing projection to predict IPS in this more challenging contention scenario. While this would not change the complexity of profiling, it would prolong it by a factor of $c$.

Another factor we have not considered in this work is application phase changes. While we deem this potentially beneficial to efficiency, we think that it is orthogonal as it does not fundamentally change the approach: if a phase change detection mechanism is in place, a phase change can be treated by our proposed approach, as a termination of an application, and a simultaneous spawn of another, with different performance characteristics. Additionally, each application phase (rather than each whole application) should then be offline profiled and characterized, which strengthens the importance of having a scalable profiling strategy. Phase change detection can be performed by observing the online-monitored performance measurements and detecting significant changes. DyPO [9] proposes a way to implement phase change detection.

Last, a limitation of our current implementation is the ability to migrate applications only at specific times. Each application consists of a basic loop that iterates a number of times decided by our event generator (see Section 5). Migrations can happen only between iterations of this loop-if a migration is decided, the iteration currently running will start over on the new core. This actually punishes the efficiency of Heuristic 3 and also minimizes the effectiveness of trying to migrate applications more often: Calling Heuristic 3 in regular intervals, instead of only spawns and terminations, achieves earlier migration of some applications on a more efficient core, but the original version of the heuristic eventually does the same. As our focus is on providing an efficient initial response, the corrective action capabilities of Heuristic 3 are already sufficient to remedy any bad decisions coming from model inaccuracy or reduced core availability. The challenge of identifying the optimal migration frequency has been studied in Reference [27].

\section{CONCLUSIONS}

In this article, we propose a runtime manager for single-ISA, heterogeneous, DVFS-capable CMPs aiming to improve energy efficiency. The manager uses a prediction model to estimate performance and power for possible configurations and a heuristic to choose candidate solutions. Our prediction model estimates application performance using scalable profiling of applications that can run on the system and projecting this information to the current system status, as measured through online monitoring. Subsequently, it uses these performance estimations to predict system 
power with an analytical model based only on the specific platform's characteristics. Through exhaustive evaluation, we found our model to mispredict performance, power and energy efficiency by an average $2.5 \%, 6.1 \%$, and $6 \%$, respectively. We have designed three alternative heuristics, the execution time of which grows sublinearly with respect to the system size. Guided by an oracle model, our heuristics are able to select a configuration only $2.5 \%$ less efficient than a brute-force, exhaustive approach. Our approach as a whole (model and heuristics) was evaluated with a plethora of dynamic, multi-application scenarios. Comparing to widely used governors, when not considering individual applications' performance requirements, our solution was 3\% more energy efficient than the powersave governor and twice as energy efficient compared to the other governors. Additionally, our approach is able to support applications with performance requirements at the cost of $18 \%$ lower energy efficiency versus the powersave governor, which, however, misses the performance targets by $23 \%$. Furthermore, it maintains an efficiency advantage of $52 \%$ and $58 \%$ over the interactive and ondemand governors, respectively, which can satisfy all requirements.

\section{REFERENCES}

[1] Ali Aalsaud, Rishad Shafik, Ashur Rafiev, Fie Xia, Sheng Yang, and Alex Yakovlev. 2016. Power-aware performance adaptation of concurrent applications in heterogeneous many-core systems. In Proceedings of the International Symposium on Low Power Electronics and Design (ISLPED'16). ACM, New York, NY, 368-373.

[2] Arunachalam Annamalai, Rance Rodrigues, Israel Koren, and Sandip Kundu. 2013. An opportunistic prediction-based thread scheduling to maximize throughput/watt in AMPs. In Proceedings of the 22nd International Conference on Parallel Architectures and Compilation Techniques (PACT'13). Piscataway, NJ, 63-72.

[3] L. Benini, A. Bogliolo, and G. De Micheli. 2000. A survey of design techniques for system-level dynamic power management. IEEE Trans. Very Large Scale Integr. Syst. 8 , 3 (June 2000), 299-316.

[4] R. Cochran, C. Hankendi, A. K. Coskun, and S. Reda. 2011. Pack amp; Cap: Adaptive DVFS and thread packing under power caps. In Proceedings of the 44th Annual IEEE/ACM International Symposium on Microarchitecture (MICRO'11). 175-185.

[5] Daniele De Sensi, Massimo Torquati, and Marco Danelutto. 2016. A reconfiguration algorithm for power-aware parallel applications. ACM Trans. Archit. Code Optim. 13, 4, Article 43 (Dec. 2016).

[6] Christina Delimitrou and Christos Kozyrakis. 2013. Paragon: QoS-aware scheduling for heterogeneous datacenters. In Proceedings of the 18th International Conference on Architectural Support for Programming Languages and Operating Systems (ASPLOS'13). 77-88.

[7] Bryan Donyanavard, Tiago Mück, Santanu Sarma, and Nikil Dutt. 2016. SPARTA: Runtime task allocation for energy efficient heterogeneous many-cores. In Proceedings of the 11th IEEE/ACM/IFIP International Conference on Hardware/Software Codesign and System Synthesis (CODES'16). ACM, New York, NY.

[8] Bhavishya Goel. 2016. Measurement, Modeling, and Characterization for Energy-efficient Computing. Chalmers University of Technology and Göteborg University.

[9] Ujjwal Gupta, Chetan Arvind Patil, Ganapati Bhat, Prabhat Mishra, and Umit Y. Ogras. 2017. DyPO: Dynamic paretooptimal configuration selection for heterogeneous MpSoCs. ACM Trans. Embed. Comput. Syst. 16, 5s, Article 123 (Sept. 2017).

[10] M. R. Guthaus, J. S. Ringenberg, D. Ernst, T. M. Austin, T. Mudge, and R. B. Brown. 2001. MiBench: A free, commercially representative embedded benchmark suite. In Proceedings of the IEEE International Workshop on Workload Conference (WWC'01). 3-14.

[11] Marcus Hähnel and Hermann Härtig. 2014. Heterogeneity by the numbers: A study of the ODROID XU+E big. LITTLE platform. In Proceedings of the 6th USENIX Conference on Power-Aware Computing and Systems (HotPower'14). USENIX Association, Berkeley, CA, 3-3.

[12] S. Herbert and D. Marculescu. 2007. Analysis of dynamic voltage/frequency scaling in chip-multiprocessors. In Proceedings of the International Symposium on Low Power Electronics and Design (ISLPED'07). 38-43.

[13] Rakesh Kumar, Keith I. Farkas, Norman P. Jouppi, Parthasarathy Ranganathan, and Dean M. Tullsen. 2003. SingleISA heterogeneous multi-core architectures: The potential for processor power reduction. In Proceedings of the 36th Annual IEEE/ACM International Symposium on Microarchitecture (MICRO'03). IEEE Computer Society, Washington, DC.

[14] G. Liu, J. Park, and D. Marculescu. 2013. Dynamic thread mapping for high-performance, power-efficient heterogeneous many-core systems. In Proceedings of the IEEE 31st International Conference on Computer Design (ICCD'13). $54-61$. 
[15] NASA. [n.d.]. NAS Benchmarks. Retrieved from https://www.nas.nasa.gov/publications/npb.html.

[16] V. Pallipadi, S. Li, and A. Belay. 2016. cpuidle-Do nothing, efficiently. In Proceedings of the Linux Symposium (Vol. 2). 119-125.

[17] A. Pathania, S. Pagani, M. Shafique, and J. Henkel. 2015. Power management for mobile games on asymmetric multicores. In Proceedings of the IEEE/ACM International Symposium on Low Power Electronics and Design (ISLPED'15). 243-248.

[18] M. Pricopi, T. S. Muthukaruppan, V. Venkataramani, T. Mitra, and S. Vishin. 2013. Power-performance modeling on asymmetric multi-cores. In Proceedings of the International Conference on Compilers, Architecture and Synthesis for Embedded Systems (CASES'13). 1-10.

[19] Qualcomm. [n.d.]. Qualcomm snapdragon 808 processor. Retrieved from https://www.qualcomm.com/products/ snapdragon/processors/808.

[20] Samsung. [n.d.]. ODROID XU3. Retrieved from https://www.hardkernel.com/main/products/prdt_info.php?g_code= g140448267127\&tab_idx=2.

[21] Samsung. [n.d.]. Samsung Exynos. Retrieved from http://www.samsung.com/semiconductor/minisite/exynos/.

[22] S. Sarma, T. Muck, L. A. D. Bathen, N. Dutt, and A. Nicolau. 2015. SmartBalance: A sensing-driven linux load balancer for energy efficiency of heterogeneous MPSoCs. In Proceedings of the 52nd ACM/EDAC/IEEE Design Automation Conference (DAC'15). 1-6.

[23] A. K. Singh, M. Shafique, A. Kumar, and J. Henkel. 2013. Mapping on multi/many-core systems: Survey of current and emerging trends. In Proceedings of the 50th ACM/EDAC/IEEE Design Automation Conference (DAC'13). 1-10.

[24] E. Del Sozzo, G. C. Durelli, E. M. G. Trainiti, A. Miele, M. D. Santambrogio, and C. Bolchini. 2016. Workload-aware power optimization strategy for asymmetric multiprocessors. In Proceedings of the Conference on Design, Automation and Test in Europe (DATE'16). EDA Consortium, San Jose, CA, 531-534.

[25] Texas Instruments. [n.d.]. INA231 sensor. Retrieved from http://www.ti.com/lit/ds/symlink/ina231.pdf.

[26] E. Vasilakis, I. Sourdis, V. Papaefstathiou, A. Psathakis, and M. G. H. Katevenis. 2017. Modeling energy-performance tradeoffs in ARM big.LITTLE architectures. In Proceedings of the 27th International Symposium on Power and Timing Modeling, Optimization and Simulation (PATMOS'17). 1-8.

[27] Ilias Vougioukas, Andreas Sandberg, Stephan Diestelhorst, Bashir M. Al-Hashimi, and Geoff V. Merrett. 2017. Nucleus: Finding the sharing limit of heterogeneous cores. ACM Trans. Embed. Comput. Syst. 16, 5s, Article 152 (Sept. 2017). DOI : https://doi.org/10.1145/3126544

[28] Y. Zhu and V. J. Reddi. 2013. High-performance and energy-efficient mobile web browsing on big/little systems. In Proceedings of the IEEE 19th International Symposium on High Performance Computer Architecture (HPCA'13). 13-24.

Received September 2018; revised October 2018; accepted November 2018 